\title{
Erratum to : La conjecture de non réalisation due à N. Kuhn
}

\section{Lionel Schwartz}

Published online: 3 August 2010

(C) Springer-Verlag 2010

\section{Erratum to : Invent math (1998) 134 :211-227 DOI $10.1007 / \mathrm{s} 002220050263$}

Je remercie Gérald Gaudens de m'avoir signalé une erreur concernant le cas d'un nombre premier impair (section 3 de l'article, pages 224 à 227) dans l'article porté en référence.

Il n'y a aucune raison évidente pour que l'homomorphisme de Bockstein soit trivial dans la cohomologie du plan projectif $C$ considéré page 226, lignes 21 à 23, alors que cet espace avait été introduit dans ce but. La démonstration du théorème 3.1 ne fonctionne donc que si l'on suppose que l'homorphisme de Bockstein est nul dans le module qui apparaît dans l'énoncé. En ajoutant cette dernière hypothèse on obtient, par la méthode utilisée pour $p=2$, un théorème analogue à ceux de [2], sans recours à la théorie de l'invariant de Hopf 1.

La démonstration évoquée ci-dessus peut être réparée dans certains cas significatifs (voir [5]). Cependant, pour établir le résultat en toute généralité, il convient de procéder autrement; c'est ce qui est fait dans [1] où l'on donne un traitement unifié des cas $p=2$ et $p>2$.

The online version of the original article can be found under doi : $10.1007 / \mathrm{s} 002220050263$.

L. Schwartz $(\bowtie)$

Départment de Mathématiques, Université de Paris Nord, URA 742 du CNRS,

Avenue Jean-Baptiste Clément, 93430, Villetaneuse, France

e-mail: schwartz@math.univ-paris13.fr 
N. Kuhn a proposé depuis [3] une autre méthode pour $p=2$. Cette méthode est basée sur la tour de Goodwillie-Arone, elle améliore un peu les résultats de [4]. Mais, selon $M$. Stelzer, elle semble faire face à une difficulté analogue à celle de [4] quand on cherche à l'étendre à $p>2$.

\section{Bibliographie}

1. Applications depuis $K(\mathbb{Z} / p, 2)$ et une conjecture de N. Kuhn. HAL-00476377, http://arxiv.org/abs/1005.0567; ou pour une version plus récente avec quelques corrections mineures. http://zeus.math.univ-paris13.fr/schwartz/Publi/EML.pdf

2. Kuhn, N.: On topologically realizing modules over the Steenrod algebra. Ann. Math. 141, 321-347 (1995)

3. Kuhn, N.: Topological nonrealization results via the Goodwillie tower approach to iterated loopspace homology. Algebraic Geom. Topol. 8, 2109-2129 (2008)

4. Schwartz, L.: A propos de la conjecture de non-réalisation due à N. Kuhn. Invent. Math. 134, 211-227 (1998)

5. Schwartz, L.: A propos de l'homomorphisme de Bockstein : un exemple de non réalisibilité. http://zeus.math.univ-paris13.fr/ schwartz/Publi/Bockstein.pdf 